\title{
Évolution des systèmes de gestion des connaissances et d'intelligence économique : d'une revue de la littérature scientifique anglophone à l'exploration de points de vue francophones
}

\author{
Stéphane Goria \\ Université de Lorraine, CREM, Nancy, France \\ stephane.goria@univ-lorraine.fr
}

\begin{abstract}
Au cours des années 1980-1990s les concepts de gestion des connaissances et d'intelligence économique ont été progressivement formalisés, puis transposés en systèmes dédiés au début des années 2000s (Goria, 2006). Au-delà des débats concernant les définitions qui ont pu être proposées, ces systèmes peuvent être simplement considérés comme des dispositifs fondés sur la mise en œuvre partielle ou totale de pratiques d'intelligence économique ou de gestion des connaissances. Dans cet ordre d'idées, il y a une quinzaine d'années, lorsqu'ils étaient dits d'intelligence économique, ces systèmes avaient pour objectif de soutenir la stratégie d'une organisation et leur spectre de mise en application comprenait des services de : veille, protection du patrimoine informationnel, réseautage, influence et lobbying (Salles, 2003). Lorsqu'ils étaient dits de gestion des connaissances, ils avaient généralement pour objectif l'amélioration durable des performances et capacités d'innovation de l'organisation en s'appuyant sur des fonctions d'acquisition, de cartographie, de capitalisation, de partage et de création de connaissances (Ermine, 2003).
\end{abstract}

Tels que, jusqu'en 2004, ces systèmes ont rencontré un certain succès auprès des entreprises et autres institutions, sans que leur conception ne change radicalement. C'est alors que la révolution numérique du Web 2.0 a eu lieu (Legrenzi, 2010). Progressivement il est devenu de plus en plus important pour les organisations d'intégrer les réseaux sociaux dans leur stratégie (Montargot \& Duyck, 2017). Ainsi, des réseaux sociaux d'entreprises ont été directement intégrés aux systèmes de gestion des connaissances et d'intelligence économique créant de la sorte une nouvelle génération de projets de knowledge management nommés KM 2.0 (Mounoud \& Dudezert, 2008) et d'intelligence économique 2.0 (Quoniam \& Lucien, 2009). De même, depuis cette époque l'intérêt des organisations pour le traitement de données hétérogènes et massives s'est plus que développé. On peut même désormais considérer l'intégration du Big Data dans les systèmes des entreprises comme une évolution logique des systèmes d'intelligence économique seuls (Mercanti-Guérin, 2013) ou associés à un système à base de connaissances (Sybord, 2015). Par conséquent, il semble normal qu'il y ait eu aussi des changements dans les pratiques des usagers impactés par l'apparition de ces «nouvelles » technologies. Ces changements ont pu ensuite influencer la conception et les considérations des systèmes d'intelligence économique comme de gestion des connaissances (Carlier, 2013). Maintenant qu'une douzaine d'années s'est écoulée depuis l'émergence du Web 2.0 et la démocratisation des technologies qui lui sont associées, au-delà de la conception technique pure de ces systèmes, un certain nombre de questions peuvent être posées. L'une qui nous intéresse tout particulièrement concerne l'évolution du regard des chercheurs à propos de ces systèmes en tant qu'objet de recherche. Les considèrent-ils toujours de la même manière ? Comment leurs considérations ont-elles évolué ?

Nous proposons de donner dans cette introduction quelques éléments de réponses à ces questions à l'aide d'une analyse bibliométrique des écrits scientifiques anglophones référencés dans les bases de données ACM Digital Library et $W$ eb of Science complétée d'une présentation des autres articles de ce numéro. Cette 
analyse n'est pas fondée sur un traitement en profondeur des écrits produits à propos des systèmes de gestion des connaissances et d'intelligence économique, mais est centrée sur le vocabulaire employé dans les titres d'un ensemble de publications scientifiques si rapportant. Nous partons ainsi de l'hypothèse que si des éléments nouveaux en lien avec la recherche prennent suffisamment d'importance, alors ils finissent par être présents de manière récurrente dans les titres des articles, chapitres de livres et autres ouvrages scientifiques.

\section{Éléments méthodologiques de l'analyse bibliométrique}

D'un point de vue scientifique et anglophone, il nous a semblé intéressant d'observer comment les recherches à propos des systèmes d'intelligence économique ou de gestion des connaissances avaient évolué. Pour ce faire, nous avons interrogé deux portails d'accès à des bases de données scientifiques: ACM Digital Library et Web of Science. Le premier référence plus particulièrement des actes de conférences en lien avec les technologies de l'information et le second des publications dans des revues scientifiques tous domaines confondus. A priori, leur complémentarité pour une couverture de la littérature scientifique anglophone nous a semblé suffisante pour une analyse de l'évolution des écrits portant sur les systèmes d'intelligence économique ou de gestion des connaissances. D'autres auteurs ont d'ailleurs utilisé cette complémentarité entre ces deux mêmes ressources informationnelles pour réaliser une approche bibliométrique centrée sur les systèmes d'information (Kosar et al, 2016). Nous avons aussi complété cette interrogation par une interrogation des portails Cairn, Revue.org et $\mathrm{Hal}$ afin d'estimer de manière purement quantitative la production francophone par rapport à l'anglophone.

Nous avons limité notre analyse du corpus de textes anglophones à l'étude seule des variations de vocabulaire dans les titres des écrits scientifiques que nous obtiendrions en réponses. Ce corpus de titres a été constitué à partir de requêtes ciblant des écrits employant deux expressions dont l'une se rapporte à celle de « système » et l'autre à celle d' "intelligence économique » ou de "gestion des connaissances ». Les champs interrogés ont été : title et topic ${ }^{l}$ pour le portail $W$ eb of Science et, title et abstract pour le portail $A C M$ Digital Library. Ainsi, nous avons choisi d'associer à l'expression «système ", les expressions anglaises : « system » et "platform $»^{2}$. Puis, nous avons traduit l'expression française "gestion des connaissances » par celle de "knowledge management»; traduction qui en règle générale ne suscite pas de débat. Enfin, concernant l'expression "intelligence économique», nous avons choisi de l'associer à trois expressions anglophones pouvant y faire référence : «competitive intelligence », «strategic intelligence» et «business intelligence». Même si «business intelligence » est une expression ambigüe, car elle peut aussi se traduire en français par l'expression « analyse décisionnelle » (Gabillaud, 2009) ou celle d' « informatique décisionnelle » (Foucard, 2014), nous l'avons gardée, car certains auteurs l'emploi au sens d' «intelligence économique » (Gaidelis, 2013 ; Pluchart, 2008) et que le nombre de références obtenues avec cette seule expression, via les deux portails scientifiques, n'a pas été trop important (414 occurrences pour douze années), ce qui permettait de filtrer ensuite par lecture des résumés une partie au moins des résultats non pertinents (273 titres sur les 414 ont été ainsi retenus). Nous verrons d'ailleurs, dans ce même numéro, avec la traduction de l'article de (Erickson \& Rothberg, 2017) que désormais la plupart des outils de business intelligence sont plus ou moins associés à des systèmes de gestion des connaissances. À ce titre, même si leur traduction n'est pas associée à celle d'intelligence économique, elle peut l'être toutefois à celle de gestion des connaissances, ce qui justifie l'intégration des articles employant ce terme dans le corpus analysé.

\footnotetext{
${ }^{1}$ Le portail Web of Sciences ne permet pas d'interroger seulement les résumés. Nous avons donc utilisé à défaut, comme champs complémentaire à celui de title, celui de topic.
}

${ }^{2}$ Les versions au pluriel de ses expressions ont aussi été prises en comptes. 
Pour ce qui concerne la partie technique de cette analyse quantitative, nous avons d'abord utilisé par la plateforme Endnote pour exporter et dédoublonner les données de ces deux portails (ACM Digital Library et Web of Sciences). Au total nous avons obtenu 2793 titres d'écrits scientifiques uniques à partir de nos requêtes. Pour traiter ces derniers, nous avons fait appel au logiciel Tropes et l'interface Iramuteq qui est une extension du logiciel d'analyse statistique R. Iramuteq nous a permis d'étudier le corpus dans son intégralité en mettant en avant les rapprochements par cooccurrences des termes employés dans les titres. Après différents essais, nous avons développé notre analyse avec ce logiciel à partir de dendrogrammes à six classes générés à partir de notre corpus divisé en trois sous-corpus relatifs à trois périodes de temps : 2005-2008, 2009-2012 et 2013-2016. Ces dendrogrammes sont des arbres hiérarchiques (figure 2) qui regroupent des termes en fonction d'un calcul de proximité lié à leur fréquence de cooccurrences dans les titres collectés. Un groupe de termes de cette structure est appelé classe et, les classes sont agrégées deux à deux, toujours selon le degré de proximité de l'ensemble des termes qu'elles contiennent. Les pourcentages associés à la représentation d'une classe représentent l'importance de chaque classe vis-à-vis de l'ensemble du vocabulaire traité. Les mots les plus importants, c'est-à-dire les plus fréquents figurent, par ordre d'importance, juste en dessous de l'intitulé de la classe. Il nous a semblé que ce type de présentation exprimait bien certains changements de vocabulaire et de répartition de ces derniers dans les titres analysés (figure 3).

Pour compléter ces informations et surtout approfondir notre compréhension de l'évolution du vocabulaire des titres, nous avons utilisé le logiciel Tropes. Ce logiciel permet de montrer, notamment, le positionnement des mots les plus fréquents et cooccurrents avec des expressions choisies ${ }^{3}$. Dans la logique de notre sélection d'écrits scientifiques, nous avons pris comme terme central de ces analyses les différents vocabulaires employés pour obtenir nos titres de textes: «system» et "platform », "knowledge management», "business intelligence », «competitive intelligence 》 et "strategic intelligence». Finalement, seules les analyses centrées sur les expressions «system » et «platform» ont permis de mettre en évidence quelques changements notables de vocabulaire.

\section{Principales observations}

De manière générale, une analyse quantitative très simplifiée nous montre le nombre de publications dédiées aux systèmes de gestion des connaissances représente une très large majorité des publications (oscillant entre 84 et 95\% de l'ensemble des publications du corpus) et que les titres des publications pouvant se rapportés à la fois aux systèmes de gestion des connaissances et d'intelligence économique représente un effectif quasi constant, mais marginal (il ne dépasse jamais les $2.5 \%$ de l'ensemble des publications d'une année). Il y a eu apparemment un pic de productions scientifiques entre 2008 et 2010, mais en explorant ce sous-corpus spécifique, nous n'avons pas trouvé de changement de vocabulaire spécifique par rapport à la période 2005-2007 ; seul le nombre des occurrences des termes déjà présents a augmenté (voir figure 4 et 5).

\footnotetext{
${ }^{3}$ Avec Tropes l'ordre des mots dans une phrase est conservé. Pour une expression donnée, les termes qui lui sont fréquemment associés sont considérés selon qu'ils se trouvent à droite ou à gauche du terme ciblé par l'analyse. Le sens de lecture est indiqué par une flèche présente sous les graphes générés par ce logiciel.
} 

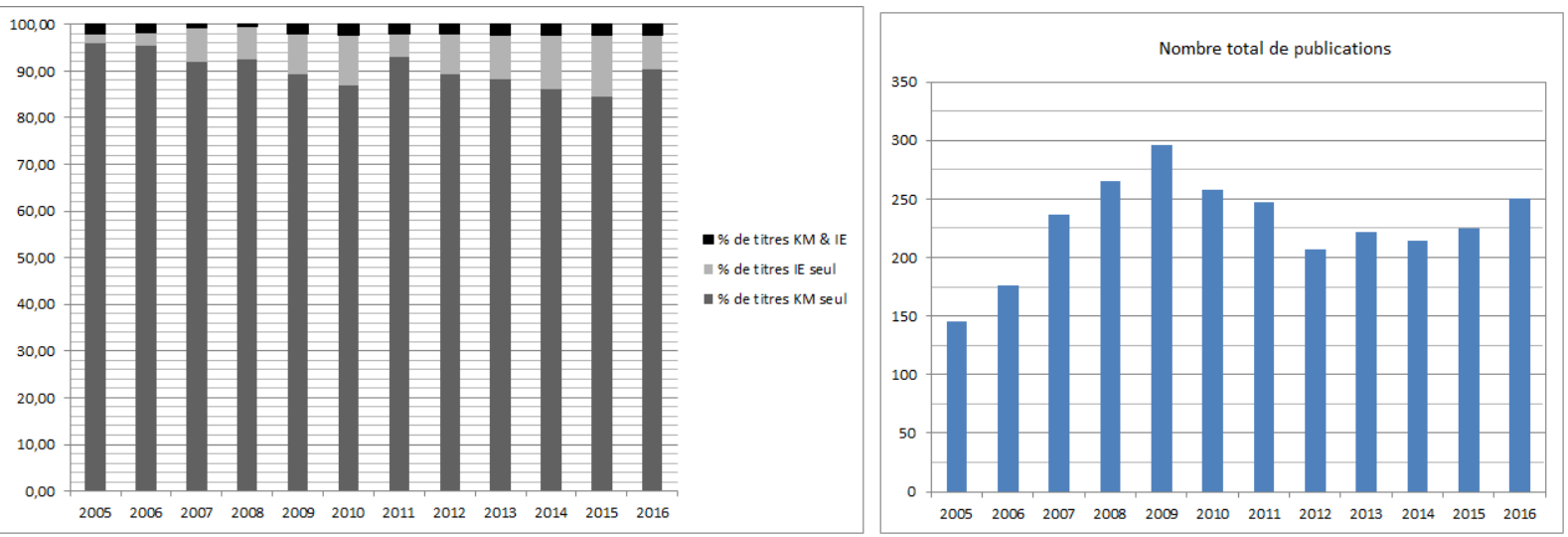

Figure 1. Évolution du nombre total de publications (à droite) et des pourcentages (à gauche) de représentativité des écrits à propos de systèmes ou plateformes pouvant se rapporter uniquement à la gestion des connaissances (KM), à l'intelligence économique (IE) ou aux deux à la fois.

Après avoir pris connaissance de ces premières observations, nous avons souhaité comparer rapidement cette production anglophone de publications relatives aux systèmes de gestions des connaissances ou d'intelligence économique par rapport à la francophone. Pour ce faire, nous avons choisi les portails d'accès aux publications scientifiques Cairn, Revue.org et Hal et y avons interrogé les champs résumés et titre.
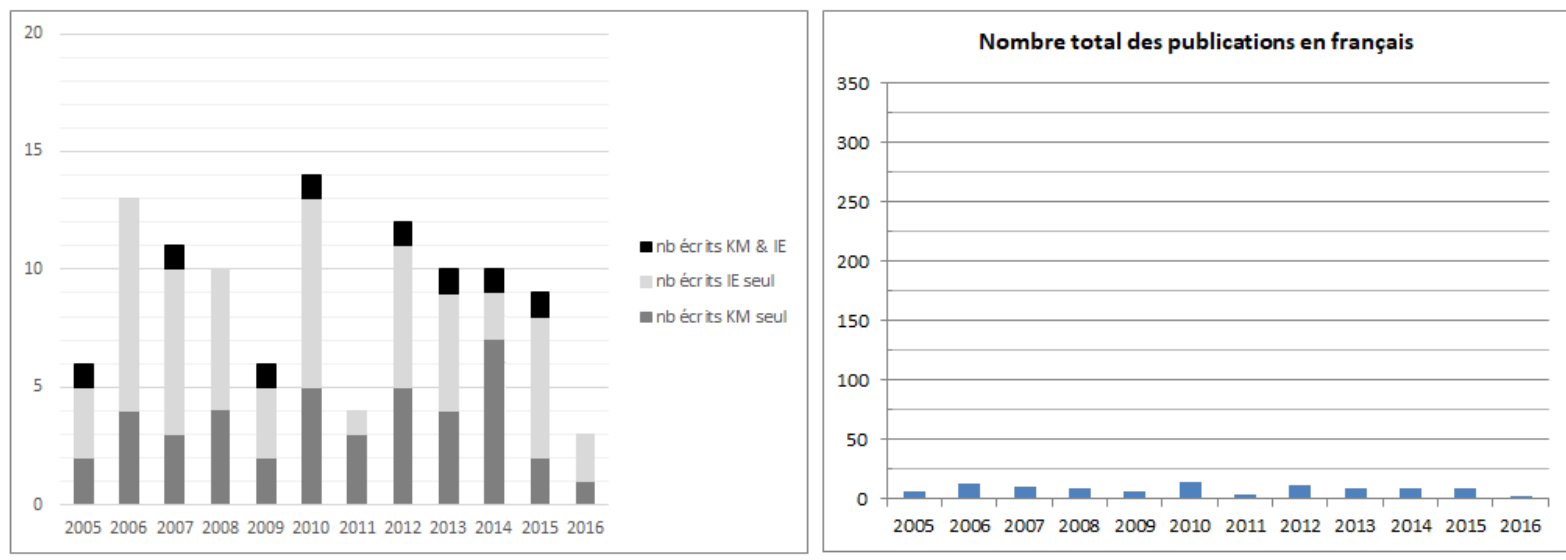

Figure 2. Évolution du nombre total de publications (à droite) et quantité d'écrits (à gauche) à propos de systèmes ou plateformes pouvant se rapporter uniquement à la gestion des connaissances $(\mathrm{KM})$, à l'intelligence économique (IE) ou aux deux à la fois

Au total pour les années 2005 à 2016, nous avons obtenu 108 publications (articles, actes de conférences, actes de colloque, chapitres de livre, livres). La figure 2 représente le total des publications obtenues par année (à droite) sur la même échelle que pour les publications anglophones. Le nombre des publications étant bien moindre, nous avons affiché sur la gauche de la figure 2 les quantités d'articles de relatives à la gestion des connaissances (KM), à l'intelligence économique (IE) ou aux deux à la fois. Les expressions que nous avons combinées pour obtenir ces publications étaient: pour l'intelligence économique « intelligence économique», «intelligence compétitive», «veille stratégique», pour la gestion des connaissances, "gestion des connaissances », "gestion de connaissances », " gestion de la connaissance », «management de la connaissance », «management de connaissances», «management des connaissances, «knowledge management» et pour système, «système» et "plateforme». On peut constater que les publications faisant référence à la fois à l'intelligence économique, à la gestion des connaissances et à un système sont au plus d'une par année. De plus, par rapport aux publications anglophones, ce sont les publications que l'on peut rattacher aux systèmes et à l'intelligence économique qui sont les plus 
nombreuses à l'exception des années 2011 et 2014. Toutefois, le faible nombre des publications francophones (avec une moyenne de 9 publications par an) rend très sensible le rapport existant entre les publications plutôt dédiées aux systèmes et à l'intelligence économique vis-à-vis de celles se rapporta aux systèmes et à la gestion des connaissances. Ce rapport est en effet au plus de 5 publications supplémentaires du côté de l'intelligence économique (en 2006) et de 5 publications supplémentaires du côté de la gestion des connaissances (2014).

Pour ce qui l'analyse du corpus de titres d'articles anglophones, l'analyse à l'aide de dendrogrammes (figure 3) montre l'apparition, dans les titres des écrits de 2013-2016 par rapport à ceux de 2005-2008, des termes media et social dans une même classe (classe 1 du dendrogramme de droite). Toutefois, comme nous l'avons dit plus haut, suite au développement des technologies en lien avec le Web 2.0, nous nous y attendions. Ceci dit, cette classe permet de constater aussi que ces mêmes termes sont associés avec une forte occurrence des termes tool et planning.

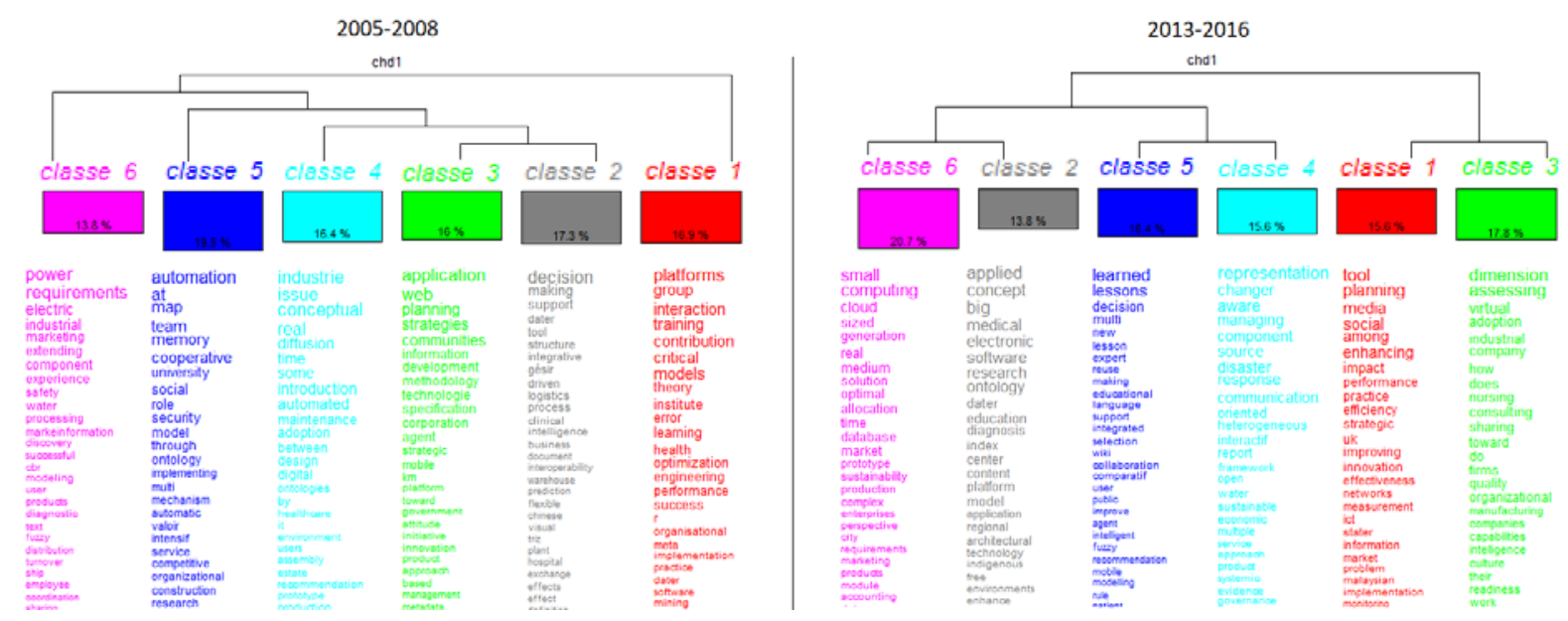

Figure 3. Total 2005-2008 (à gauche) et 2013-2016 (à droite)

La classe 2 (du dendrogramme de droite) est aussi intéressante, même si elle regroupe, en fait, deux sousclasses. D'une part elle rassemble des termes se rapportant à l'application des systèmes au domaine médical : applied, medical et diagnosis. D'autre part, cette classe comprend aussi les termes big et concept. Ceci semble simplement être lié au fait que l'expression big data est très souvent employée avec le terme concept qui lui se retrouve assez fréquemment avec les termes medical et applied. Les classes 3 et 4 ne sont pas très parlantes en termes d'évolution de vocabulaire. Si l'on résume à l'essentiel ce que l'on peut en dire ; virtual apparait dans les titres de la classe 3 liés l'implantation des systèmes dans les entreprises et communautés et, les termes relatifs aux changements de représentations (representation, changer) sont bien plus fréquents dans les dernières publications. La classe 5 (du dendrogramme de droite) quant à elle nous semble un peu plus intéressante. Elle pourrait correspondre à l'évolution de la classe 2 du corpus 2005-2008 qui est dédiée aux titres employant l'expression decision making support et qui semble intégrer désormais des éléments relatifs à l'apprentissage des systèmes ou des individus : learning, lessons, expert. Comme elle est associée en premier lieu avec la classe 4 pour les années 2013-2016, on note uen complémentarité dans les écrits traitant de conception des systèmes concernés. Enfin, dans la classe 6 (du dendrogramme de droite), ce sont les termes de cloud et computing en référence au «cloud computing» qui sont bien présents, mais aussi les termes small et medium, le plus souvent référent aux petites et moyennes entreprises. En effet, après une lecture ciblée des titres employant ces adjectifs, nous avons constaté qu'ils étaient le plus souvent associés aux termes enterprise, company, organization et business. Une autre classe intéressante de vocabulaires est celle qui regroupe des écrits portant, en grande partie, sur «la gestion des plans catastrophe ». On y retrouve souvent les termes representation, changer, managing, component, source, disaster et response. 
Lorsque nous avons analysé à l'aide du logiciel Tropes, le positionnement des cooccurrences de termes et expressions par rapport au terme system, nous avons remarqué que peu de vocabulaires nouveaux étaient apparus au cours des dernières années. Nous notons tout de même l'apparition parmi les expressions récurrentes, celles de : similarity, data system, implementation et performance (graphique en bas de la figure 4).

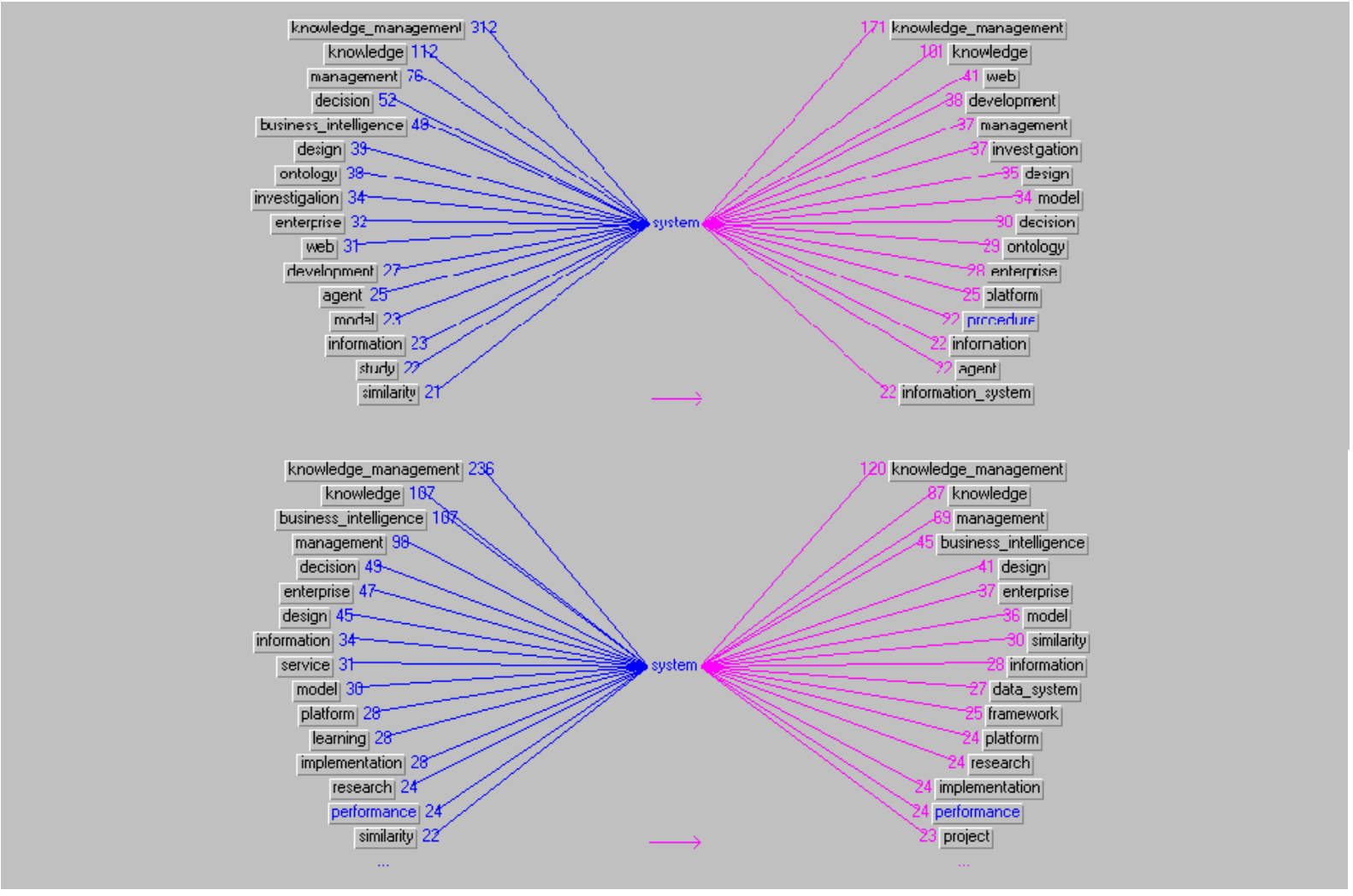

Figure 4. Comparaison des termes fréquents cooccurrents au tour de celui de system pour les corpus de titres pour les années 2005-2008 (an haut) et 2013-16 (en bas)

Pour ce qui concerne les autres termes et expressions que nous avons ciblés, seul celui de platform (figure 5) permet de mettre en évidence quelques changements, même si les fréquences d'emploi des mots sont bien moindres que dans le cas de system (figure 3). Au mois, nous constatons que les expressions e-learning et expert-system semblent avoir disparu au profit de celles de big data et de data system. 


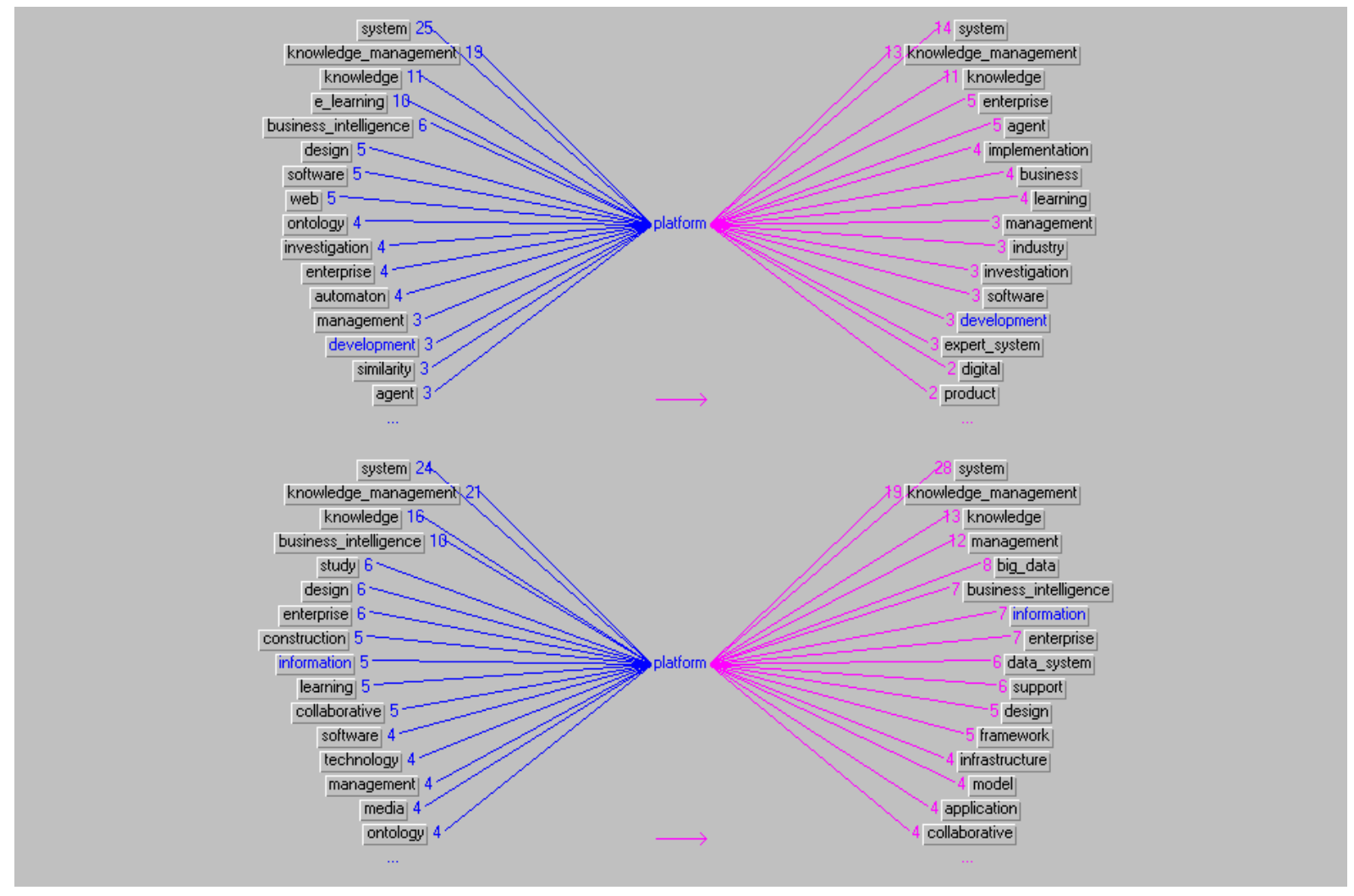

Figure 5. Comparaison des termes fréquents cooccurrents au tour de celui de platfom pour les corpus de titres pour les années 2005-2008 (an haut) et 2013-16 (en bas)

Finalement cette petite analyse bibliométrique nous a permis de conforté le constat de l'apparition du w

Résumés :

Maher SLOUMA -> communautés et visions francophones du KM

AUDREY DE CEGLIE \& NADIA HASSANI -> réseaux sociaux pro francophones femmes -> KM

Joanne du Hommet, Madjid Ihadjadene, Luc Grivel -> KM en entreprise Jeux vidéo

NDJOCK FLEUR NADINE -> IT = IE +KM -> système éducatif Cameroun

Texte sur le Big data et le KM - Competitive intelligence

Jean Sébastien Vayre -> Machines et Big data, apprentissage machines : comment les machines apprennent

Analyse :

progression (2009-2016) : learning, performance, data system, implementation 
Maher SLOUMA ; Vers une approche incrémentale du Knowledge Management. Le propos de cet article est d'étudier l'aspect évolutif et incrémental d'une démarche Knowledge Management dans les organisations à l'aide d'un pluralisme méthodologique. Dans un premier temps, il s'agit d'identifier les communautés francophones du Knowledge Management sur le Web via une étude netnographique. Les résultats obtenus serviront de base pour une étude quantitative par questionnaire en ligne. Enfin, une étude qualitative sur les questions semi-ouvertes gagnera à étayer les résultats des deux études précédentes. Au début de cette recherche, nous nous sommes posé une question principale qui se rapporte au caractère évolutif et incrémental d'une démarche Knowledge Management. Cette question a été formulée après une longue observation au sein de la communauté Knowledge Management. Cette observation a été majoritairement non participante, l'objectif était d'élaborer une problématique qui répond aux attentes et aux préoccupations de la communauté Knowledge Management. Pour ce faire, nous avons sollicité plusieurs sources d'informations. Il s'agit (1) des informations recueillies du Web, (2) des informations issues d'un questionnaire en ligne et (3) des témoignages de type qualitatif via des questions semi-ouvertes. Par la suite, nous avons testé les sept sous hypothèses de l'aspect incrémental du Knowledge Management à l'aide des données issues du questionnaire et le test de Khi-deux. Trois d'entre elles sont validées, avec des valeurs significatives : (1) le degré de perception de l'utilité du KM dans l'organisation, (2) l'innovation dans l'organisation et (3) l'évolution des étapes de la démarche KM. Nous pouvons donc affirmer que la démarche Knowledge Management est incrémentale au moins pour l'échantillon d'entreprises sur lequel nous avons travaillé.

AUDREY DE CEGLIE \& NADIA HASSANI ; Les réseaux professionnels de leaders: un nouvel outil de gestion des connaissances. Dans les entreprises, l'imposition des quotas de femmes leaders au sein des conseils d'administration à fait émerger de nombreux réseaux sociaux professionnels. Nous observerons comment les stratégies de communication se construisent en cours d'actions et comment les rapports sociaux et les savoirs communs émergent. En nous attachant plus au " Knowing », nous mettrons l'accent sur le processus de production des connaissances. Les réseaux sociaux professionnels, comme par exemple ici facebook, semblent être des outils de médiation intéressants, car ils présentent des implications différentes selon les membres. Les interactions et les informations qui s'y développent peuvent être des outils pour le management des individus. Si certains restent de simple diffuseur d'information professionnelle, d'autres semblent être des lieux d'échange où se construit un espace collaboratif producteur de connaissances. Cependant ils ne paraissent être qu'une partie visible de l'iceberg.

Joanne du Hommet, Madjid Ihadjadene, Luc Grivel; Web 2.0 et partage de l'information et des connaissances au sein d'une entreprise multinationale (Ubisoft). Nous étudions dans cet article un aspect particulier de la réception des dispositifs de gestion de connaissances en nous focalisant particulièrement sur les pratiques de partage et diffusion de l'information des cadres d'une entreprise mondiale de jeux vidéo. Les dispositifs du Web 2.0 adaptés en entreprise apparaissent désormais comme l'outil d'une vision institutionnelle qui consiste à placer l'usager au cœur de l'innovation. Nous avons pu quantifier et analyser la place prépondérante de l'humain dans les systèmes informationnels de l'entreprise. Nous avons proposé une nouvelle typologie et montré la diversité et la complémentarité des dispositifs utilisés notamment ceux issus du Web 2.0. Il est difficile d'isoler un outil (type réseau social) des autres sources et dispositifs informationnels.

NDJOCK FLEUR NADINE; la conception d'un système d'aide à la décision et le poids de l'économie informelle dans la collecte de l'information. Le processus d'intelligence économique dans la conception d'un système d'information permet de canaliser la procédure de collecte, de traitement, de stockage et de diffusion de l'information dans le but de rendre l'outil efficace quant à l'atteinte des objectifs fixés. 
L'objectif principal étant la réduction de la part d'incertitude dans la prise de décision par le moyen d'une représentation synthétique desdites informations. Mais, alors que le résultat de l'outil est évalué par rapport à l'objectif à atteindre (la prise de décision par l'utilisateur), la pertinence de la procédure suivie pour la collecte de l'information dans le processus de conception de l'outil est rarement appréciée parce que complexe. Bien souvent, les informations servant à la conception d'un système d'information sont des données déjà existantes en interne ou en externe dans les bases et les banques de données officielles (organismes internationaux et ministériels, document de travail, statistiques nationales...). Cependant, nous pensons que dans un environnement comme le système éducatif du Cameroun où le secteur informel est important, se limiter aux seules données macro des différentes sources officielles serait réducteur de la performance de l'outil. Nous proposons dans une perspective d'intelligence territoriale, de prendre en compte des données micro au niveau très local (villages, quartiers...) pour que le système d'information reflète le mieux possible la réalité de son environnement. Le développement des outils d'aide à la décision comme l'observatoire ouvre de nouvelles perspectives pour assurer la traçabilité, la fiabilité et la pertinence de l'information tout en mettant en place de nouvelles pratiques impliquant macro-informationnelle dans la construction du système d'information. Ceci nous contraint dans un certain sens d'insister sur la notion de " partage » de l'information induite par le concept du Knowledge Management (KM) (Nonaka \& Konno , 1999) dans un contexte où la discrétion et la méfiance sont de rigueur. Si les individus interagissent dans le cadre d'une mise en place d'un système d'aide à la décision, les données intégrées ne peuvent qu'être pertinentes et conduire à la performance du système.

Jean Sébastien Vayre; Machines intelligentes et économie numérique : Étude du cas d'un agent artificiel dans le domaine du travail relationnel marchand. Les technologies d'apprentissage artificiel (i.e. : machine learning) sont au coeur du mouvement big data que nous connaissons aujourd'hui. Elles suscitent beaucoup d'enthousiasme de la part des managers de l'entreprise. Car, dans le monde de l'industrie et à la manière de l'automation chez Pierre Naville (1961), les agents artificiels ont pour fonction d'automatiser une partie du travail inférentiel afférant traditionnellement aux professionnels de la gestion. Afin de mieux comprendre comment les agents artificiels peuvent participer au travail relationnel marchand à l'ère du numérique, nous proposons dans cet article d'étudier ce que les machines peuvent apprendre. Pour ce faire, nous examinons le cas concret d'un agent artificiel mobilisé par plus d'une cinquantaine d'ecommerçants. Cette contribution doit en ce sens être considérée comme une première exploration ayant pour but de défricher le vaste terrain de recherche que recouvre l'étude des agents artificiels et l'examen des conséquences humaines et sociales de leur intégration au sein des sphères de l'organisation et du marché. Car, bien que nous soyons loin de certains récits de science-fiction, il est fortement probable que, à l'image de l'automation de P. Naville (1961), les agents artificiels que nous concevons aujourd'hui préparent une nouvelle "nouvelle société industrielle ", ou plutôt informationnelle, dans laquelle la connaissance et la maitrise des processus d'interaction homme-machine constituent un enjeu important compte tenu des effets de contrôle et d'asymétrie associés à leur mise en œuvre.

\section{Bibliographie}

Carlier A. (2013). Knowledge Management et Web 2.0: Outils, méthodes et applications. Lavoisier.

Ermine, J. L. (2003). La gestion des connaissances. Hermes Lavoisier.

Foucard, J. L. (2014). La Boîte à outils du Pilote des Systèmes d'Information(2e éd). Dunod. 
Gabillaud, J. (2009). SQL Server 2008: Administration d'une base de données avec SQL Server Management Studio. Editions ENI.

Gaidelys, V. (2013). The opportunities of the use of competitive intelligence in business: literature review. Journal of Small Business and Entrepreneurship Development, 1(2), pp. 9-16.

Goria, S. (2006). Knowledge management et intelligence économique : deux notions aux passés proches et aux futurs complémentaires. Informations, Savoirs, Décisions et Médiations (ISDM), (27), 1-16.

Kosar, T., Bohra, S., \& Mernik, M. (2016). Domain-specific languages: A systematic mapping study. Information and Software Technology, 71, 77-91.

Legrenzi, C. (2010). Le Web 2.0 et la suite... ou le Web 2.0, moteur de la transformation de la société. Annales des Mines-Réalités industrielles, No. 4, pp. 60-66

Mercanti-Guérin, M. (2013). L'amélioration du reciblage par les Big Data: une aide à la décision qui menace l'image des marques?. Revue internationale d'intelligence économique, 5(2), 153-165.

Montargot, N., \& Duyck, J. Y. (2017). Les conditions d'émergence et d'accélération des réseaux sociaux numériques d'entreprises: le cas du groupe d'assurances MMA. Management \& Avenir, (1), 87-113.

Mounoud E., Dudezert A. (2008). Défis et opportunités : le KM entre technologies, comportements et organisations, in Audrélie Dudezert et Imed Boughzala, Vers le KM 2.0: Quel management des connaissances imaginer pour faire face aux défis futurs, Vuibert, Paris, pp. 179-198.

Pluchart, J. J. (2008). Le changement organisationnel des entreprises de la net-économie. La Revue des Sciences de Gestion, (6), 15-26.

Quoniam L. et Lucien A. (2009). L’intelligence économique 2.0 ? Competitive intelligence 2.0 ? Les Cabiers du Numérique, 5 (4), pp. 11-38.

Salles, M. (2003). Stratégies des PME et intelligence économique. Editions Economica.

Sybord, C. (2015). Intelligence économique et système d'aide à la décision: de l'opérationnel «big data» au stratégique «little knowledge». Revue internationale d'intelligence économique, 7(1), 83-100. 